\title{
Cost-effectiveness analysis of pembrolizumab plus chemotherapy as first-line therapy for extensive-stage small-cell lung cancer in the United States
}

\author{
Qiao Liu 1, Xia Luo 1, Liubao Peng 1, Lidan Yi 1, Xiaomin Wan 1, Jianhe Li 1, Xiaohui Zeng 2,*, \\ Chongqing Tan 1,*
}

1 Department of Pharmacy, the Second Xiangya Hospital of Central South University, Changsha, Hunan, People's Republic of China; 515735044@qq.com, luoxia@csu.edu.cn, pengliubao@163.com, Yilidan229@163.com, wanxiaomin@csu.edu.cn, lijianhexy@126.com, tanchongqing@csu.edu.cn

2 PET-CT Center, the Second Xiangya Hospital of Central South University, Changsha, Hunan, People's Republic of China; zengxiaohui2008@csu.edu.cn

* Correspondence: Xiaohui Zeng. PET-CT Center, the Second Xiangya Hospital of Central South University, Changsha, Hunan 410011, China. Tel: +8613787139446. Email: zengxiaohui2008@csu.edu.cn; Chongqing Tan. Department of Pharmacy, The Second Xiangya Hospital, Central South University, Changsha, Hunan 410011, China. Tel: +86073185292094. Email: tanchongqing@csu.edu.cn.

Simple Summary: The phase III KEYNOTE-604 study support a long-term survival benefit of pembrolizumab plus chemotherapy in the first-line treatment of extensive-stage small-cell lung cancer(ES-SCLC) than chemotherapy alone. Integrated the clinical benefits of pembrolizumab and its high cost into account, we developed a Markov model to compared the cost-effectiveness of adding pembrolizumab to standard first-line etoposide-platinum(EP) for patients with ES-SCLC from the the US payer perspective. Clinical efficacy, treatment utilization and safety data were pooled from the KEYNOTE-604 trial. Utilities were obtained from published resources. Costs were mainly collected from Medicare in 2020.In conclusion, we suggested the first-line treatment for ES-SCLC with pembrolizumab plus EP was not cost-effective compare with chemotherapy alone at a willingness-to-pay threshold of \$100 000 per QALY in the United States. Although pembrolizumab combination chemotherapy was beneficial to the survival of ES-SCLC, price reduction may be the necessary measure to improve its cost-effectiveness.

\begin{abstract}
:
Background: The phase III KEYNOTE-604 study confirmed the benefit of pembrolizumab combined with chemotherapy in the first-line treatment of extensive-stage small-cell lung cancer(ES-SCLC). Intergrated the clinical benefits of pembrolizumab and its high cost into account, this study aim to assess the cost-effectiveness of adding pembrolizumab to standard first-line etoposide-platinum (EP) for patients with ES-SCLC from the the US payer perspective. Methods: A Markov model was developed to compared the costs and quality-adjusted life-years (QALYs) of pembrolizumab plus EP and placebo plus EP over a 10-year time horizon. Clinical efficacy, treatment utilization and safety data were pooled from the KEYNOTE-604 trial. Utilities were obtained from published resources. Costs were mainly collected from Medicare in 2020. Sensitivity analyses were performed to examined the robustness of our model. Results: Adding pembrolizumab to standard first-line EP, resulted in better effectiveness than the use of EP alone for ES-SCLC by 0.22 QALYs. Pembrolizumab
\end{abstract}


plus EP was dominated economically by placebo plus EP, leading to an incremental cost-effectiveness ratio(ICER) of $\$ 334,373$ / QALY. Deterministic sensitivity analyses indicated that the uncertainty in model parameters exerts no substantial effect on our results. Probability sensitivity analysis indicated that probabilities for pembrolizumab plus EP being cost-effective within a wide rang of willingness to pay were modest. Conclusion: From the US payer perspective, the first-line treatment for ES-SCLC with pembrolizumab plus EP was not cost-effective compare with placebo plus EP. Although pembrolizumab combination chemotherapy was beneficial to the survival of ES-SCLC, price reduction may be the necessary measure to improve its cost-effectiveness.

Keywords:cost-effectiveness; pembrolizumab; etoposide-platinum; extensive-stage small-cell lung cancer; small cell lung cancer.

\section{Introduction}

Small-cell lung cancer(SCLC) is a highly malignant pulmonary tumor and characterized by high proliferation, great invasion, and priority of early distant metastases[1-2]. Extensive-stage SCLC (ES-SCLC) approximately composes $2 / 3$ of all diagnosed cases of SCLC[3]. Although the majority of ES-SCLC occur mutation of TP53(90\%) and RB1(65\%), the target driven gene is not clear, which leads to limited progress in the treatment of ES-SCLC[4]. In the pre-immunotherapy era, platinum based(carboplatin or cisplatin) etoposide chemotherapy has always been the standard-of-care first-line treatment for ES-SCLC[5-7], which is associated with poor outcomes (a 5 -year survival rate of $6 \%-7 \%$ and median over survival(OS) of approximately10 months) [8-9]. More efficacious first-line therapy is urgently needed to improve this prognosis.

In recently years, Immune checkpoint inhibitors(ICIs) has demonstrated promising antitumor activity in patient with ES-SCLC, including as first-line treatments[10-12]. Pembrolizumab is a humanized IgG4 monoclonal antibody that targets PD-1 pathway and restores T-cell immune activity[13]. In October 2016, pembrolizumab become the first PD-1 ICI approved for first-line use for patients with non small cell lung cancer(NSCLC)[14].Shortly afterward, the US Food and Drug Administration(FDA) has successively approved new indications for pembrolizumab,both as monotherapy and in combination with platinum and pemetrexed, but still confined to patients with advanced or metastatic NSCLC[14-15].Shortly thereafter in June 2019, the FDA granted accelerated approvel to pembrolizumab as third-line or later therapy for patients with metastatic SCLC due to its durable response rate and a manageable safety profile[16], which marks its position in the field of SCLC. Recently, a phase III KEYNOTE-604 clinical trial assessed the efficacy and safety of adding pembrolizumab to standard first-line etoposide-platinum(EP) for ES-SCLC[12].The results revealed that pembrolizumab plus EP significantly extended progression-free survival (PFS)(hazard ratio [HR],0.75; 95\% CI, 0.61 to $0.91 ; \mathrm{P}=0.0026$ ), while also prolonged OS(HR, $0.80 ; 95 \% \mathrm{CI}, 0.64$ to $0.98 ; \mathrm{P}$ 5 .0164) than EP chemotherapy alone[12]. Besides, safety profiles of pembrolizumab plus EP were as expected, with no new or unexpected toxicities observed[12]. There data support the value of pembrolizumab as first-line use for this historically difficult-to-treat cancer.

Give that the new efficacy evidence for pembrolizumab and its expensive cost, cost-effectiveness analysis comparing pembrolizumab combined standard first-line chemotherapy EP with EP alone was worth discussed. Previous study suggested that adding ICIs to standard first-line chemotherapy may not be considered as a cost-effective choice for ES-SCLC[17-18]. 
However, the cost-effectiveness of the most recently reported first-line ICI option- pembrolizumab, has yet to be investigated. Hence,we are interested to explore whether this new combination therapy provide clinical benefit at a acceptable cost for patients with ES-SCLC from the US payer perspective.

\section{Results}

Based on the our basic cost-effectiveness analysis for patients with previously untreated ES-SCLC over a 10-year time horizon(Table 1), incremental costs associated with the first-line use of pembrolizumab plus EP versus placebo plus EP were $\$ 87,017(\$ 146,873$ vs $\$ 59,286)$, and primarily comprise of drug acquisition costs for first-line treatment, followed by drug acquisition costs for second-line treatment and AE management costs for first-line treatment. Incremental effectiveness associated with the first-line use of pembrolizumab plus EP versus placebo plus EP were 0.22 QALYs (1.01 QALYs vs 0.79 QALYs). Overall, first-line pembrolizumab plus EP produced an ICER of $\$ 364,373$ per QALY compared to placebo plus EP.

Table 1. Summary base case results

\begin{tabular}{lccc}
\hline Outcomes & Pembrolizumab plus EP & Placebo plus EP & Difference \\
\hline LYs & 1.43 & 1.13 & 0.3 \\
$\quad$ PFS state & 0.57 & 0.45 & 0.12 \\
$\quad$ PD state & 0.86 & 0.68 & 0.18 \\
QALYs & 1.01 & 0.79 & 0.22 \\
$\quad$ PFS state & 0.46 & 0.35 & 0.11 \\
$\quad$ PD state & 0.55 & 0.44 & 0.11 \\
Costs,\$US & 126362 & 44890 & 81472 \\
1st-line drug acquisition costs & 94384 & 3600 & 90784 \\
2nd-line drug acquisition costs & 1439 & 614 & 825 \\
Drug administration cost & 8680 & 8111 & 569 \\
AEs management (first-line) & 1250 & 978 & 272 \\
AEs management(second-line) & 13572 & 23180 & -9608 \\
Follow-up visit & 3482 & 4612 & -1130 \\
Supportive care & 817 & 607 & 210 \\
Death cost & 2738 & 3188 & -451
\end{tabular}

ICER,\$US

Per LY 
Per QALY

334,373

EP, etoposide-platinum.; LY, life-year;QALY, quality-adjusted life-year;PFS, progression-free survival; PS, progression survival;ICER, incremental cost-effectiveness ratio. 


\subsection{Sensitivity Analyses}

The tornado diagram depicted in Figure 1 reported the results of DSA. The ICER between pembrolizumab plus EP and placebo plus EP decreased significantly with the decrease of pembrolizumab price per mg and the utility of 1 month prior to death. Other parameters had smaller influence on the ICER for pembrolizumab plus EP vs placebo plus EP. In general, within the upper and lower limits of model parameters, the ICER fluctuated in the range of $\$ 28,0000$ per QALY and $\$ 46,0000$ per QALY, which were far higher than the WTP threshold of $\$ 100,000$ per QALY in our model.

The cost-effectiveness acceptability curve depicted in Figure 2 reported the cost-effectiveness probability of pembrolizumab plus EP under different WTP thresholds. Compared with placebo plus EP, pembrolizumab plus EP had a $1.55 \%$ probability being cost-effective at the WTP threshold of $\$ 100,000$ per QALY. When the WTP threshold increased to $\$ 210,000$ per QALY, pembrolizumab plus EP had a $50 \%$ probability being cost-effective. In addition, we also analyzed the cost-effectiveness acceptability of pembrolizumab plus EP under different pembrolizumab price (Supplementary Figure S1).Compared with placebo plus EP, pembrolizumab plus EP had a probability of over $50 \%$ being cost-effective, when the price of pembrolizumab per mg less than $\$ 10$.

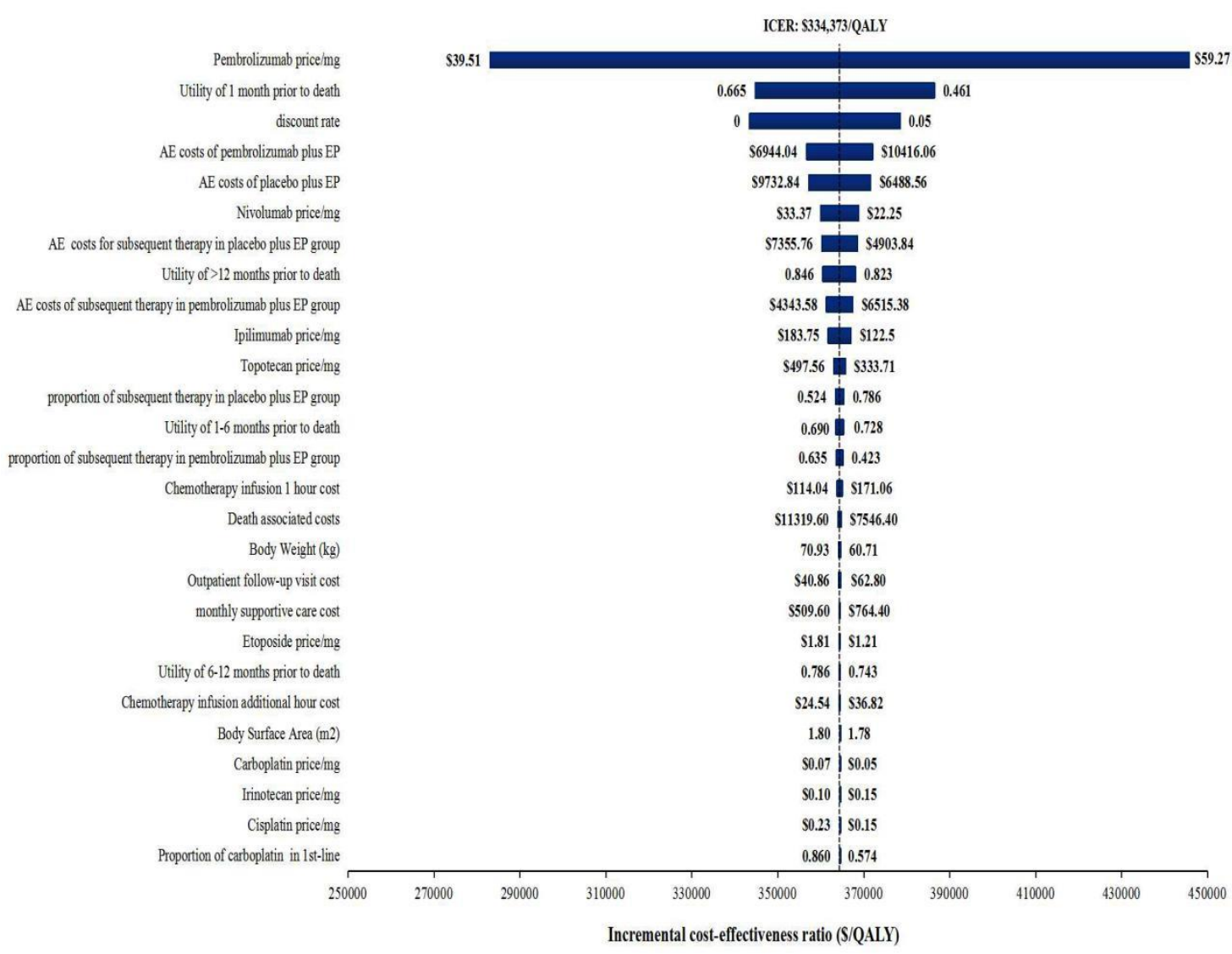

Figure 1. The results of Deterministic Sensitivity Analysis. The black dotted line represents the the ICER of $\$ 364374$ per quality-adjusted life-year (QALY) from the base case results. The The ranges for each model parameters listed represents the lower and upper bounds used in the sensitivity analysis.ICER indicated incremental cost-effectiveness ratio; EP, etoposide-platinum. 


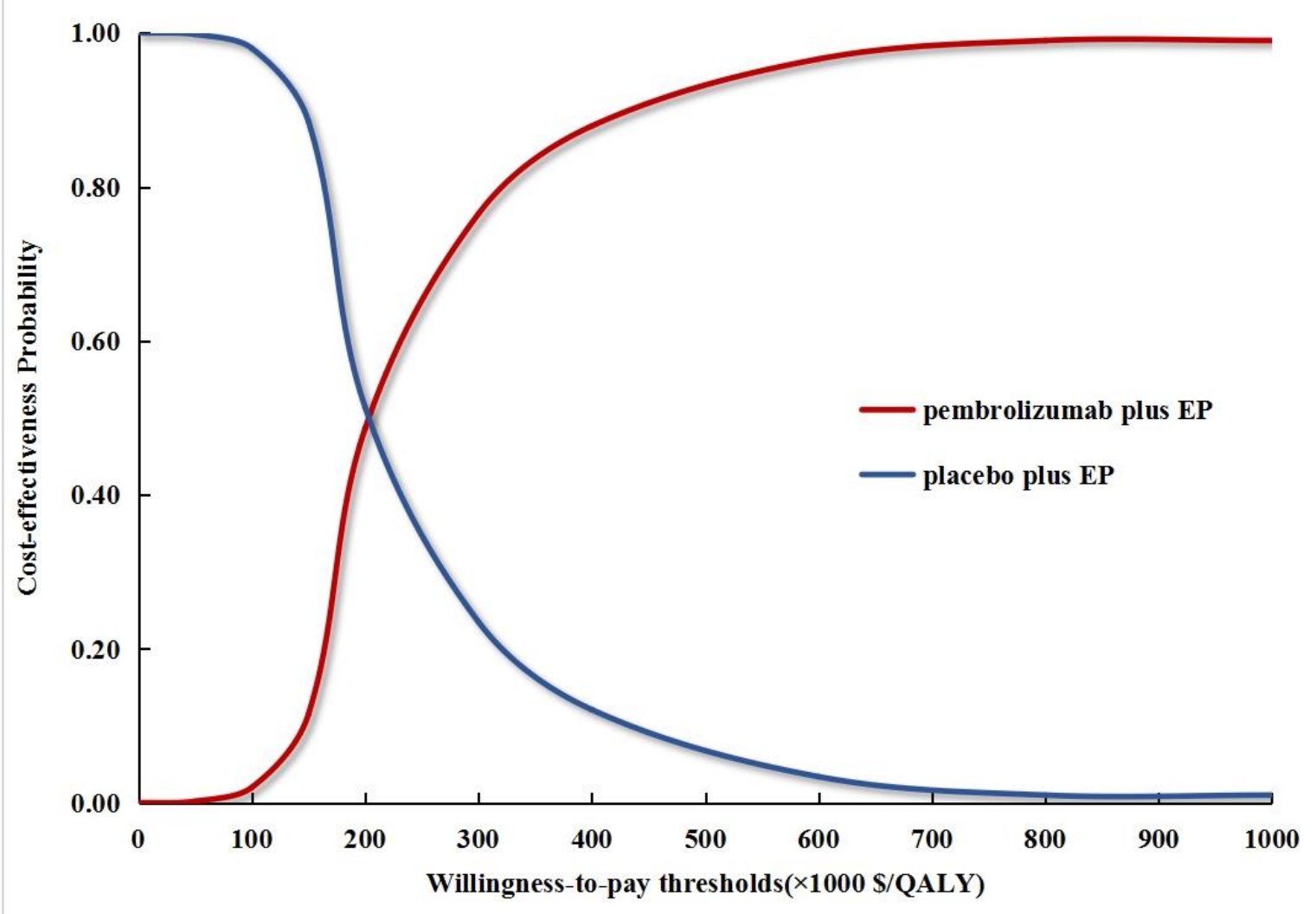

Figure 2. The cost-effectiveness acceptability curve. The red curve indicated the probability of pembrolizumab plus EP being cost-effective against placebo plus EP under different willingness-to-pay (WTP) thresholds.QALY indicated quality-adjusted life-year; EP, etoposide-platinum.

\section{Discussion}

First-line treatment options for ES-SCLC are usually limited to conventional chemotherapy, until the approval of atezolizumab or durvalumab combined with chemotherapy in recent years[10-11]. A latest phase III clinical study, KEYNOTE-604, compared the star ICI, pembrolizumab plus EP with placebo plus EP as the first-line treatment for ES-SCLC [12]. Preliminary results from the KEYNOTE-604 trail were mixed. Pembrolizumab plus EP did provide clinical benefit in the first-line treatment for ES-SCLC, however the median OS narrowly missed the prespecified significance threshold [12]. Given that medians do not fully capture the PFS and OS benefits of immuotherapy[19], and the EYNOTE-604 KM curves support a long-term benefit of pembrolizumab plus EP[12], as well as the price gap between pembrolizumab plus EP and EP, it is significant for us to evaluate its cost-effectiveness from the US payer perspective by simulating the long-term survival of patients with ES-SCLC in KEYNOTE-604 trial through Markov model.

On the basis of our results, the addition of pembrolizumab to first-line EP chemotherapy produced an ICER of $\$ 334,373$ per QALY, which beyond the WTP threshold of $\$ 100,000$ per QALY. As a result, pembrolizumab plus EP was not considered to be a cost-effective first-line treatment for patients with ES-SCLC from the US payer perspective. DSA exhibited that the most influential 
parameter of our model was the price of pembrolizumab. Low price of pembrolizumab would lower the total cost of pembrolizumab plus EP, therefore,leading to a decrease in the ICER. We found that the pembrolizumab plus EP would produce an ICER lower than the WTP threshold when the reduction in the price of pembrolizumab exceeded $65 \%$. Another parameter that has a great influence on our model is the utility of 1 month prior to death. Due to the lack of published health utility value data for ES-SCLC patients, utility values used in this analysis were therefore refer to NSCLC patients[20]. To illustrate the influence of health utility values on the model, each utility value was defined a variable range in sensitivity analyses. The results indicate that the upper and lower limits of utility values failed to allow pembrolizumab plus EP reach an ICER below the WTP threshold.

In view of the relatively new clinical evidence of first-line application of immunotherapy in ES-SCLC, its economic evaluation were rarely reported. There are only two published cost-effectiveness analyses for immunotherapy combined with chemotherapy as frist-line treatment for ES-SCLC, both from an American perspective. [17-18]. One established a partition survival model to compared durvalumab plus EP versus EP for patients with previously untreated ES-SCLC, and found that durvalumab plus EP was associated with an ICER of $\$ 355,488$ per QALY. The other study developed a Markov model to assess the cost-effectiveness of atezolizumab plus chemotherapy, and reported an ICER of $\$ 528,810$ per QALY. Although these two previous studies and our current study concluded that the combination of immunotherapy and chemotherapy was not a cost-effective choice in the first-line treatment of ES-SCLC, pembrolizumab combined with chemotherapy strategy seemed to provide a more effective balance between incremental cost and quality-adjusted survival gained than was atezolizumab or durvalumab.

Strengths of our study are worth highlighting. First, the cost and clinical outcomes associated with pembrolizumab plus EP and placebo plus EP over a 10-year time horizon were estimated through economic modeling. In our model, clinical efficacy, treatment utilization and safety data were pooled from the KEYNOTE-604 trial, and costs were mainly collected from Medicare in 2020. Compared with conventional meta-analytic techniques, a Markov model was able to provide more accurate and reliable long-term projection of cost and effectiveness. Second, the dose sizes and infusion timing for subsequent therapy in our model were consistent with the recommendations of NCCN guidelines[21], which my be closer to real clinical practice. Third, the cost of first-line pembrolizumab plus EP treatment for 7 median cycles (range,1-35 cycles) were considered in our model to avoid the influence of the duration of pembrolizumab on the results. As observed in the keynote-604 trial, patients discontinued treatment were not just because of disease progression, but also because of $\mathrm{AE}$, physician decision, radiographic progression, withdrawal of consent. Therefore, the median number of cycles would better reflect that patient time on first-line therapy in the keynote-604 trial. In addition to the strengths mentioned, our trail-based Markov model was able to simulate the process of treatment and survival for patients with previously untreated ES-SCLC, so as to reproduce general clinical treatment scenes. Therefore,our study is expected beneficial to doctors and health policy makers.

Certainly, our study has several limitations. First, there is inherent uncertainty in the long-term extrapolation of PFS and OS. In our model, published KM survival curves were digitized to replicate the PFS and OS data, which were not available in the keynote-604 trial. Among four most commonly 
used parametric survival functions, log-logistic distribution was chose to fit and extrapolate those replicated survival data based on statistical goodness of fit test. Although this method emulated the actual PFS and OS data observed in the keynote-604 trial, the current model could be optimized when more mature data long-term survival data are available. Second, because the keynote-604 trial did not provide the quality-of-life utility data for participant, and so far there is no published study on the health utility values for ES-SCLC patients, therefore, the health utilities used in our model was refer to that of NSCLC patients enrolled in the KEYNOTE-189 trial. This assumption was based on the similarity of treatment strategies between the two trial. Third, quite a number of subsequent therapies were available to patients who experience disease progression, based on the subsequent therapy date from the keynote-604 trial. To simplify the model, we only taken into account the common treatment strategies. However, the results of sensitivity analysis indicated that the proportion of subsequent therapy and related drug costs had a less remarkable effects on our model.

\section{Materials and Methods}

\subsection{Simulation Model}

Clinical data in our model was derived from the randomized, double-blind, phase III KEYNOTE-604 study(ClinicalTrials.gov Identifier:NCT03066778), which exempt our study from the approval of the institutional research ethics board.

Using TreeAge Pro 2018 software (TreeAge,Williamstown, Massachusetts), we established a Markov model to evaluate the long-term cost and effectiveness for patients with previously untreated ES-SCLC from the US payer perspective. Based entirely on the KEYNOTE-604 clinical trial[12], two first-line strategies were compared in our model, referred to as (1) pembrolizumab plus EP and (2) placebo plus EP The pembrolizumab plus EP group received four doses of pembrolizumab and EP, followed by maintenance of pembrolizumab up to 35 doses. The placebo plus EP group only received up to four doses of EP. With regard to the full trial population received treatment, $71.1 \%$ (317 of 446) were treated with carboplatin,and $28.9 \%$ (129 of 446) were treated with cisplatin.

After the failure of first-line treatments, as described in the KEYNOTE-604 trial, over half of the patients were treated with subsequent therapy (52.9\% in the pembrolizumab plus EP group; $65.5 \%$ in the placebo plus EP group). Nivolumab plus ipilimumab, etoposide, topotecan and irinotecan were the common subsequent therapies. In agreement with the recommendations of the National Comprehensive Cancer Network(NCCN) Guidelines, supportive care was provided to patients who did not receive subsequent therapy[21]. Table S1 in the Supplementary provides the detailed information on first and second-line treatment regimen.

Our model consists of three mutually exclusive health states, referred as progression-free survival(PFS), progressive disease (PD), and death (Figure 3).All patients entered the model in the PFS state and could move to another health state according to transition probabilities. Patients in each heath state was assigned a certain health utility and corresponding treatment, incurred a certain medical costs and health effect[quantified in the form of quality-adjusted life years(QALYs)].In this analysis, both cost and quality-adjusted life years (QALYs) were discounted at 
an annual rate of $3 \%$. Based on a consideration of therapeutic schedules and expected overall survival time of ES-SCLC, a Markov cycle length of 3 weeks and a 10-year time horizon were set in our model. The summary outcome of our model was the incremental cost-effectiveness ratio (ICER) ,calculated as the the incremental per additional QALY gained and compared with a willingness-to-pay (WTP) threshold of $\$ 100,000$ per QALY[22].
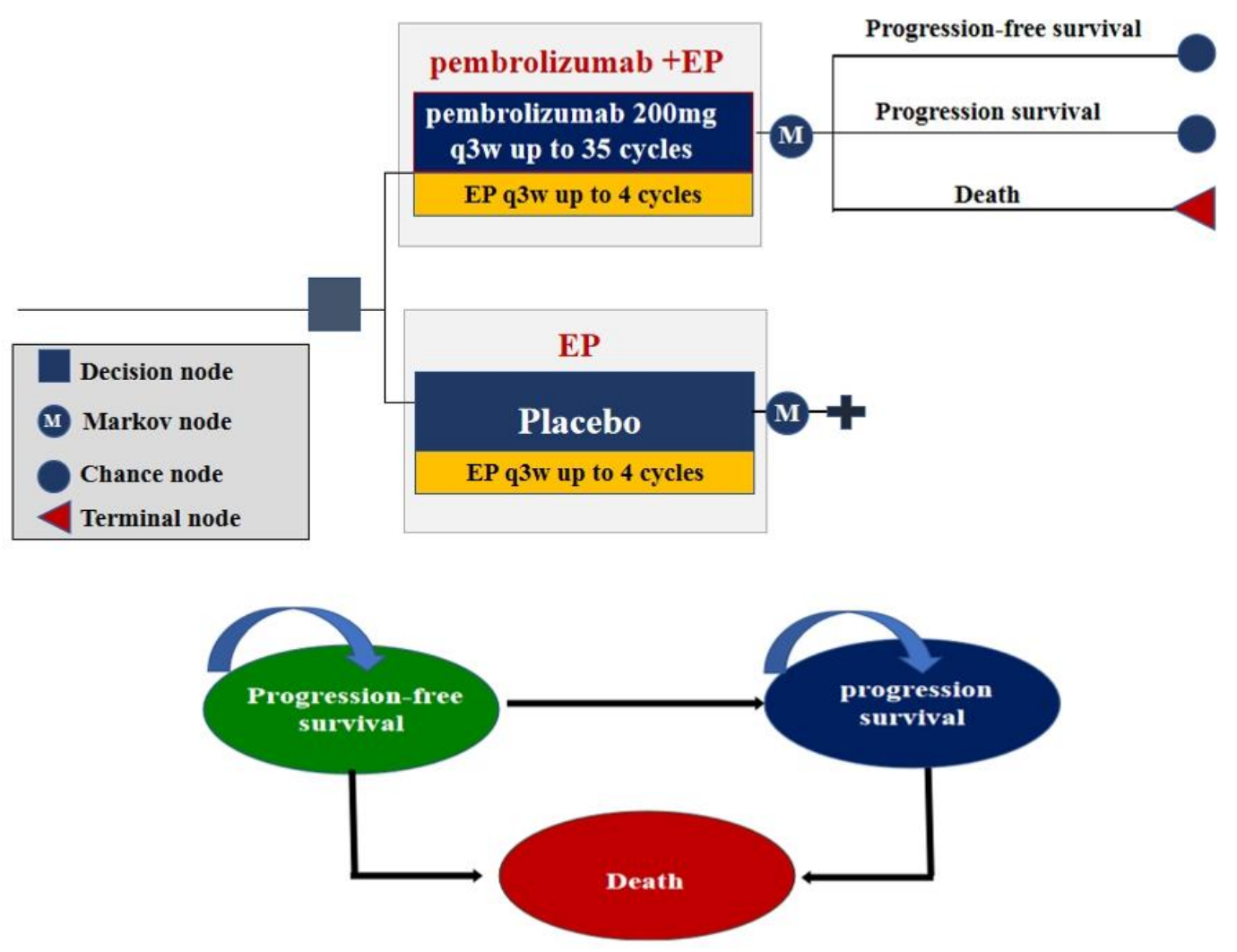

Figure 3. Markov model simulating outcomes for the patients with ES-SCLC in KEYNOTE-604 trial.The model consists of three health states: PFS, PD and death. All patients entered the model in the PFS state and were randomly assigned to receive the two fist-line treatment. During each Markov cycle, patients could move to another health state according to transition probabilities. ES-SCLC indicated extensive-stage small-cell lung cancer; EP, etoposide-platinum; PFS, progression-free survival; $\mathrm{PD}$, progressed disease

\subsection{Survival and Health State Utilities}

The Kaplan-Meier (KM) curves of OS and PFS reported in the KEYNOTE-604 trial were used for estimating the transition probability of death and disease progression from ES-SCLC treated with first-line pembrolizumab plus EP and placebo plus EP[12].Firstly, OS data points for the first 33 months and PFS data points for the first 21 mouths were extract from OS and PFS KM curves using GetData Graph Digitizer software package (version 2.25;

http://www.getdata-graphdigitizer.com/index.php), and then these data points were fitted and extrapolated by survival functions using R software (version 3.3.1, http://www.r-project.org). Secondly, by using Akaike information criterion (AIC) and Bayesian information criterion (BIC), the goodness of fit of four commonly used parametric survival distributions were tested, including 
exponential, weibull, log-normal and log-logistic.Thirdly, in view of lower AIC and BIC values indicated better fit, log-logistic distribution was chosen for this analysis(Supplementary Table S2 detailed the AIC and BIC values).Finally, the log-logistic distribution parameters, $\operatorname{theta}(\theta)$ and $\operatorname{kappa}(\gamma)$, were computed by R language(showed in table 2). For the validation of our model, the modeled survival curves were compared with the investigated KM curves (Supplementary Figure S2)

The time-dependency transition probabilities of death from ES-SCLC were calculated based on the survival function of the log-logistic distribution:

$$
t p_{\text {die }}\left(t_{u}\right)=1-\frac{1+\exp (\theta)(t-u)^{k}}{1+\exp (\theta) t^{k}}
$$

Accordingly, the time-dependency transition probabilities of PS from ES-SCLC were calculated as follows:

$$
t p_{p f s}\left(t_{u}\right)=\frac{1+\exp (\theta)(t-u)^{k}}{1+\exp (\theta) t^{k}} /\left(1-t p_{\text {die }}\right)
$$

Where the $\mathrm{u}$ is the Markov cycle, and $\mathrm{t}$ is calculated as integer multiples of the Markov cycle.

As information on quality-of-life data was not published as part of the KEYNOTE-604 trial results, model inputs for health utility values were therefore source from EuroQOL-5D (EQ-5D) 3-level utility data of patients enrolled in the KEYNOTE-189 trial[20], because this trial primary assessed pembrolizumab combined platinum-based chemotherapy exactly as the KEYNOTE-604 trial[12,23]. All information regarding utility values used in the model were provided in Table S3.

Table 2. Clinical inputs to the models

\begin{tabular}{lll}
\hline Parameter & Input values & Ref \\
\hline Log-logistic OS survival model & & \\
Pembrolizumab plus EP & $\theta=0.008564, \gamma=1.719092$ & {$[12]$} \\
placebo plus EP & $\theta=0.002564, \gamma=2.260147$ & {$[12]$} \\
Log-logistic PFS survival model & & \\
Pembrolizumab plus EP & $\theta=0.007161, \gamma=2.510618$ & [12] \\
placebo plus EP & $\theta=0.000417, \gamma=4.150059$ & [12] \\
Proportion of carboplatin treatment & 0.711 & [12] \\
Proportion of cisplatin treatment & 0.289 & {$[12]$} \\
proportion of subsequent therapy & & {$[12]$}
\end{tabular}


placebo plus EP

AE incidence of Pembrolizumab plus EP

Neutropenia

Anemia

Thrombocytopenia

Pneumonia

0.067

0.408

0.045

Neutropenia

Anemia

Thrombocytopenia
[12]

AE incidence of placebo plus EP

EP, etoposide-platinum.; AE, adverse event.

\subsection{Costs}

The collection of direct medical costs in our analysis were carried out from three aspects: regimen related costs, adverse event(AE) management costs and other disease management costs.

Regimen related costs involves drug acquisition and administration costs. The price of each drug per mg were calculated on the basis of October 2020 Average Sales Price Drug Pricing Files (version updated October 3, 2020) form the U.S. Centers for Medicare \& Medicaid Services(CMS)[24]. In calculating the dosage amounts, a base case patient with a body weight of $70.32 \mathrm{~kg}$, a body surface area of $1.79 \mathrm{~m} 2$ and creatinine clearance rate of $70 \mathrm{ml} / \mathrm{min}$ were assumed in our model[18,25]. Drug administration costs were obtained though Physician Fee Schedule Look-Up Tool form CMS[26].Statistics of adverse event management costs were based on the Healthcare Cost and Utilization Project using diagnosis Code selection for ICD-10[27]. Grade 3 or higher serious AEs with an incidence of more than $3 \%$ were considered in the model. Other disease management costs, including outpatient follow-up visit costs, supportive care costs and death-associated costs, were derived form previously published articles[18,25].All information regarding costs used in the model were listed in eTable 3.

\subsection{Sensitivity Analysis}

To test the robustness of our model with respect to uncertainty in model parameters,one-way deterministic sensitivity analyses(DSA) and probabilistic sensitivity analysis (PSA) were performed. In DSA, the influence of model parameters varying individually on the cost-effectiveness results were assessed. The plausible ranges of model parameters were derived from publish studies whenever available or $\pm 20 \%$ of base-case values. In PSA, the influence of model parameters varying simultaneously on the cost-effectiveness results were assessed. Model parameters that varied at the same time randomly sampled from prespecified distributions, which followed the recommendations 
of the ISPOR-SMDM Modeling Good Research Practice Working Group[28]. We performed PSA using a Monte Carlo simulation with 1,000 iterations to generate 1,000 ICER estimates for the two competing first-line treatments. Parameter ranges and distributions use in DSA and PSA were detailed in Table 3.

Table 3. Model parameters: baseline values, ranges, and distributions for sensitivity analysis

\begin{tabular}{|c|c|c|c|c|}
\hline parameters & value & Ranges & Distribution & Ref \\
\hline \multicolumn{5}{|l|}{ Costs } \\
\hline \multicolumn{5}{|l|}{ Regimen related costs } \\
\hline Pembrolizumab price/mg & 49.39 & $39.51-59.27$ & Gamma(100,0.2.025) & [24] \\
\hline Etoposide price/mg & 1.51 & $1.21-1.81$ & Gamma(100,66.353) & {$[24]$} \\
\hline Irinotecan price/mg & 0.06 & $0.05-0.07$ & $\begin{array}{l}\text { Gamma }(100,1732.502 \\
\text { ) }\end{array}$ & {$[24]$} \\
\hline Cisplatin price/mg & 0.19 & $0.15-0.23$ & Gamma(100,533.049) & [24] \\
\hline Nivolumab price/mg & 27.81 & $22.25-33.37$ & Gamma(100,3.596) & [24] \\
\hline Ipilimumab price/mg & 153.13 & $122.50-183.75$ & Gamma(100,0.653) & {$[24]$} \\
\hline Topotecan price/mg & 414.63 & $331.71-497.56$ & Gamma(100,0.241) & [24] \\
\hline Irinotecan price/mg & 0.12 & $0.10-0.15$ & Gamma(100,3.011) & [24] \\
\hline Chemotherapy infusion 1 hour & 142.55 & 114.04-171.06 & Gamma(100,0.702) & {$[26]$} \\
\hline $\begin{array}{l}\text { Chemotherapy infusion } \\
\text { additional hour }\end{array}$ & 30.68 & $24.54-36.82$ & Gamma(100,2.290) & {$[26]$} \\
\hline \multicolumn{5}{|l|}{ Adverse event management costs } \\
\hline 1st-line pembrolizumab plus EP & 8680.05 & 6944.04-10416.06 & Gamma(100,0.012) & [27] \\
\hline 1st-line placebo plus EP & 8110.70 & $6488.56-9732.84$ & Gamma(100,0.012) & [27] \\
\hline $\begin{array}{l}\text { subsequent therapy of } \\
\text { pembrolizumab plus EP group }\end{array}$ & 5429.48 & $4343.58-6515.38$ & Gamma(100,0.018) & [27] \\
\hline $\begin{array}{l}\text { subsequent therapy of placebo } \\
\text { plus EP group }\end{array}$ & 6129.80 & 4903.84-7355.76 & Gamma(100,0.016) & [27] \\
\hline
\end{tabular}

\section{Other disease management costs}

$\begin{array}{lcclc}\text { Outpatient follow-up visit } & 52.33 & 41.86-62.80 & \text { Gamma(100,1.911) } & \text { [18] } \\ \text { Monthly supportive care } & 637 & 509.60-764.40 & \text { Gamma(100,0.157) } & \text { [25] } \\ \text { Death associated costs } & 9433 & 7546.40-11319.60 & \text { Gamma(100,0.011) } & {[25]}\end{array}$




\begin{tabular}{|c|c|c|c|c|}
\hline \multicolumn{5}{|l|}{ Utilities } \\
\hline$>12$ months prior to death & 0.834 & $0.823-0.846$ & $\operatorname{Beta}(83.4,16.6)$ & [23] \\
\hline 6-12 months prior to death & 0.765 & $0.743-0.786$ & $\operatorname{Beta}(76.5,23.5)$ & [23] \\
\hline 1-6 months prior to death & 0.709 & $0.690-0.728$ & $\operatorname{Beta}(70.9,29.1)$ & [23] \\
\hline 1 month prior to death & 0.563 & $0.461-0.665$ & $\operatorname{Beta}(56.3,43.7)$ & [23] \\
\hline \multicolumn{5}{|l|}{ Others } \\
\hline $\begin{array}{l}\text { Proportion of carboplatin in } \\
\text { first-line treatment }\end{array}$ & 0.711 & $0.574-0.860$ & $\operatorname{Beta}(317,129)$ & [12] \\
\hline $\begin{array}{l}\text { proportion of subsequent therapy } \\
\text { in Pembrolizumab plus EP group }\end{array}$ & 0.529 & $0.423-0.635$ & $\operatorname{Beta}(118,105)$ & [12] \\
\hline $\begin{array}{l}\text { proportion of subsequent therapy } \\
\text { in placebo plus EP group }\end{array}$ & 0.655 & $0.524-0.786$ & $\operatorname{Beta}(146,77)$ & {$[12]$} \\
\hline Body Weight (kilograms) & 70.32 & $69.71-70.93$ & Gamma(100,1.422) & [25] \\
\hline Body Surface Area (meters2) & 1.79 & $1.78-1.80$ & Gamma(100,55.865) & [25] \\
\hline
\end{tabular}

EP, etoposide-platinum.

\section{Conclusions}

In the treatment of ES-SCLC, adding pembrolizumab to standard first-line chemotherapy would not be a cost-effective option from the US payer perspective. Price reductions remain the most pratical solution to balance the incremental cost and quality-adjusted survival gain for pembrolizumab combined with chemotherapy strategy.

Supplementary Materials: Supplementary File, Figure S1:The cost-effectiveness acceptability curve, Figure S2: Parametric survival distributions fitted and extrapolated, Table S1:Treatment regimens and dosage in the Model, Table S2:Survival functions fitted and extrapolated, Table S3:Cost and Utility Parameters.

Author Contributions: Ph.D. CQ. Tan, Ph.D. LB. Peng and Q. Liu contributed to the planning of the study, acquisition of data, and interpretation of results and critically reviewed/revised the manuscript. Ph.D. X.H. Zeng and X. Luo interpreted the results and critically reviewed/revised the manuscript. Ph.D. JH.Li and Ph.D. $\mathrm{XM}$. Wang contributed to the planning of the study, acquisition and analysis of the data, and interpretation of results and critically reviewed/revised the manuscript. LD.Yi contributed to the planning of the study and interpretation of results and critically reviewed/revised the manuscript. Q. liu adapted the model, conducted the analyses, interpreted the results and wrote the manuscript. All authors read and approved the final manuscript.

Funding: This work was supported by the Hunan Provincial Natural Science Foundation [grant numbers 2019JJ50864] and Scientific research project of Hunan Health Commission in 2019[grant numbers B2019156]

Acknowledgments: I certify that no individuals other than the listed co-authors contributed to this publication.

Conflicts of Interest: Qiao Liu, Chongqing Tan, Liubao Peng, Lidan Yi, Xiaomin Wan, Xiaohui Zeng and Xia Luo declare that they have no conflict of interest. 


\section{References}

1. S.N. Waqar, D. Morgensztern, Treatment advances in small cell lung cancer (SCLC), Pharmacol Ther. 180 (2017) 16-23.

2. S. Wang, J. Tang, T. Sun, et al. Survival changes in patients with small cell lung cancer and disparities between different sexes, socioeconomic statuses and ages, Sci Rep 7 (1) (2017) 1339.

3. R.L. Siegel, K.D. Miller, A. Jemal, Cancer statistics, 2019, CA Cancer J Clin 69 (1) (2019) 7-34.

4. M. Peifer, L. Fernández-Cuesta, M.L. Sos, et al. Integrative genome analyses identify key somatic driver mutations of small-cell lung cancer, Nat. Genet. 44 (10) (2012) 1104-1110.

5. M. Früh, D. De Ruysscher, S. Popat, L. Crinò, S. Peters, E. Felip. Small-cell lung cancer (SCLC): ESMO Clinical Practice Guidelines for diagnosis, treatment and follow-up, Ann. Oncol. 24 Suppl 6 (2013) i99-i105.

6. C.M. Rudin, N. Ismaila, C.L. Hann, et al. Treatment of Small-Cell Lung Cancer: American Society of Clinical Oncology Endorsement of the American College of Chest Physicians Guideline, J. Clin. Oncol. 33 (34) (2015) 4106-4111.

7. H. Akamatsu, K. Ninomiya, H. Kenmotsu, et al.The Japanese Lung Cancer Society Guideline for non-small cell lung cancer, stage IV, Int. J. Clin. Oncol. 24 (7) (2019) 731-770.

8. A.F. Farago, F.K. Keane. Current standards for clinical management of small cell lung cancer, Transl Lung Cancer Res 7 (1) (2018) 69-79.

9. G.S. Jones, K. Elimian, D.R. Baldwin, R.B. Hubbard, T.M. McKeever. A systematic review of survival following anti-cancer treatment for small cell lung cancer, Lung Cancer 141 (2020) 44-55.

10. L. Horn, A.S. Mansfield, A. Szczęsna, et al. Liu, First-Line Atezolizumab plus Chemotherapy in Extensive-Stage Small-Cell Lung Cancer, N Engl J Med 379 (23) (2018) 2220-2229.

11. L. Paz-Ares, M. Dvorkin, Y. Chen, et al.Durvalumab plus platinum-etoposide versus platinum-etoposide in first-line treatment of extensive-stage small-cell lung cancer (CASPIAN): a randomised, controlled, open-label, phase 3 trial, Lancet 394 (10212) (2019) 1929-1939.

12. C.M. Rudin, M.M. Awad, A. Navarro, et al. Pembrolizumab or Placebo Plus Etoposide and Platinum as First-Line Therapy for Extensive-Stage Small-Cell Lung Cancer: Randomized, Double-Blind, Phase III KEYNOTE-604 Study, J. Clin. Oncol. 38 (21) (2020) 2369-2379.

13. H.C. Chung, S.A. Piha-Paul, J. Lopez-Martin, et al. Pembrolizumab After Two or More Lines of Previous Therapy in Patients With Recurrent or Metastatic SCLC: Results From the KEYNOTE-028 and KEYNOTE-158 Studies, J. Thorac. Oncol. 15 (4) (2020) 618-627.

14. US Food and Drug Administration. Administration. Pembrolizumab (KEYTRUDA) checkpoint inhibitor. In, 2016.

15. US Food and Drug Administration. FDA grants regular approval for pembrolizumab in combination with chemotherapy for first-line treatment of metastatic nonsquamous NSCLC. In, 2018.

16. US Food and Drug Administration. FDA approves pembrolizumab for metastatic small cell lung cancer. In, 2020.

17. K. Zhou, J. Zhou, J. Huang, et al. Cost-effectiveness analysis of atezolizumab plus chemotherapy in the first-line treatment of extensive-stage small-cell lung cancer, Lung Cancer 130 (2019) 1-4.

18. L. Zhang, Y. Hang, M. Liu, N. Li, H. Cai. First-Line Durvalumab Plus Platinum-Etoposide Versus Platinum-Etoposide for Extensive-Stage Small-Cell Lung Cancer: A Cost-Effectiveness Analysis, Front Oncol 10 (2020) 602185. 
19. M.D. Hellmann, M.G. Kris, C.M. Rudin. Medians and Milestones in Describing the Path to Cancer Cures: Telling "Tails", JAMA Oncol 2 (2) (2016) 167-168.

20. R.P. Insinga, D.J. Vanness, J.L. Feliciano, K. Vandormael, S. Traore, T. Burke. Cost-effectiveness of pembrolizumab in combination with chemotherapy in the 1st line treatment of non-squamous NSCLC in the US, J Med Econ 21 (12) (2018) 1191-1205.

21. National Comprehensive Cancer Network. National. NCCN Clinical Practice Guidelines in Oncology:Small Cell Lung Cancer. In, 2020.

22. P.J. Neumann, J.T. Cohen, M.C. Weinstein. Updating cost-effectiveness--the curious resilience of the \$50,000-per-QALY threshold, N Engl J Med 371 (9) (2014) 796-797.

23. L. Gandhi, D. Rodríguez-Abreu, S. Gadgeel, et al. Garassino, Pembrolizumab plus Chemotherapy in Metastatic Non-Small-Cell Lung Cancer, N Engl J Med 378 (22) (2018) 2078-2092.

24. Centers for Medicare and Medicaid Services. October 2020 ASP drug pricing files. https://www.cms.gov/license/ama?file=/files/zip/october-2020-asp-pricing-file.zipl. Published October 3, 2020.Accessed October 20, 2020.

25. S.D. Criss, M.J. Mooradian, T.R. Watson, J.F. Gainor, K.L. Reynolds, C.Y. Kong. Cost-effectiveness of Atezolizumab Combination Therapy for First-Line Treatment of Metastatic Nonsquamous Non-Small Cell Lung Cancer in the United States, JAMA Netw Open 2 (9) (2019) e1911952.

26. Physician Fee Schedule Search. Centers for Medicare \& Medicaid Services; 2020. https://www.cms.gov/apps/physician-fee-schedule/search/search-criteria.aspx. Accessed October 2020.

27. Agency for Healthcare Research and Quality, U.S. Department of Health \& Human Services. Healthcare cost and utilization project. https://hcupnet.ahrq.gov. Accessed October 12, 2020.

28. A.H. Briggs, M.C. Weinstein, E.A. Fenwick, J. Karnon, M.J. Sculpher, A.D. Paltiel. Model parameter estimation and uncertainty analysis: a report of the ISPOR-SMDM Modeling Good Research Practices Task Force Working Group-6, Med. Decis. Making 32 (5) (2012) 722-732. 